\title{
Dissociating Processes Supporting Causal Perception and Causal Inference in the Brain
}

\author{
Matthew E. Roser, Jonathan A. Fugelsang, and \\ Kevin N. Dunbar \\ Dartmouth College
}

\author{
Paul M. Corballis \\ Georgia Institute of Technology
}

\author{
Michael S. Gazzaniga \\ Dartmouth College
}

\begin{abstract}
An understanding of relations between causes and effects is essential for making sense of the dynamic physical world. It has been argued that this understanding of causality depends on both perceptual and inferential components. To investigate whether causal perception and causal inference rely on common or on distinct processes, the authors tested 2 callosotomy (split-brain) patients and a group of neurologically intact participants. The authors show that the direct perception of causality and the ability to infer causality depend on different hemispheres of the divided brain. This finding implies that understanding causality is not a unitary process and that causal perception and causal inference can proceed independently.
\end{abstract}

Keywords: causality, split brain, callosotomy, cerebral asymmetry

An understanding of causal relations between moving objects is essential for making sense of and interacting with the dynamic physical world. It allows us to predict the effects of object interactions, such as collisions, and to affect goal-directed change on our physical environment. Ultimately, comprehension of fundamental rules governing causality is necessary for understanding both one's own actions (e.g., tool use; Johnson-Frey, 2003) and those of others (McClure, 1998).

Philosophers and scientists have long attempted to provide an account of how we know that one event causes another, such as one billiard ball causing another to move (Hume, 1978). In an experimental setting, interactions between moving stimuli, such as collisions, are often reported as involving causal relationships. This can occur even with very simple stimuli such as two moving balls, represented by light patches, on a computer screen. For example, if ball $A$ moves toward ball $B$, stops when it contacts ball $B$, and $B$ then moves away, the motion of ball $B$ is reported by the majority of observers to have been caused by ball $A$. This collision

Matthew E. Roser, Jonathan A. Fugelsang, Kevin N. Dunbar, and Michael S. Gazzaniga, Department of Psychological and Brain Sciences, Dartmouth College; Paul M. Corballis, School of Psychology, Georgia Institute of Technology.

Additional materials are on the Web at http://dx.doi.org/10.1037/08944105.19.5.591.supp

We thank Jeff Hamm for help with analyses and Margaret Funnell, David Turk, and Todd Handy for helpful suggestions. This research was supported by Dartmouth College and by National Institutes of Health Grant RO1 MH059825.

Jonathan A. Fugelsang is now at the Department of Psychology, University of Waterloo, Waterloo, Ontario, Canada.

Correspondence concerning this article should be addressed to Matthew E. Roser, Department of Psychological and Brain Sciences, Dartmouth College, Hanover, NH 03755-3569. E-mail: Matthew.Roser@Dartmouth .edu event has been termed the launching effect and is the best-known example of the stimuli used to investigate perceptual causality (Michotte, 1963; Scholl \& Tremoulet, 2000).

Perceptual causality describes the direct perception of causal structure from object interactions, rather than the inference of causal structure from observation and real-world knowledge. It has been proposed that simple two-dimensional displays of objects colliding evoke an illusion of causality (Michotte, 1963) that is constructed by the visual system in a manner similar to the construction of other high-level percepts such as three-dimensional object structure from motion (Scholl \& Tremoulet, 2000). Several lines of evidence support this contention.

Much research has shown that the reporting of causal structure from collision events is highly sensitive to the spatial and temporal properties of the stimulus (Hecht, 1996; Michotte, 1963; Scholl \& Tremoulet, 2000; White, 1995). The presence of a small gap or delay (incontiguity) between the two stimulus movements reduces the likelihood with which stimulus interactions are rated as causal. Furthermore, a spatially and temporally contiguous collision event, when presented for only $50 \mathrm{~ms}$, can make a noncausal event presented alongside it appear causal, suggesting that the perceptual system is able to rapidly integrate spatial and temporal information (Scholl \& Nakayama, 2002).

In addition to the stimulus-driven nature of causal reports, the observation that infants are sensitive to physical causality at only 6 months of age (Leslie \& Keeble, 1987; Oakes, 1994), and the involvement of areas of the brain responsive to visual movement (Blakemore et al., 2001), have been taken to suggest that causal structure is extracted from collision events by the visual system (Blakemore et al., 2001; Fonlupt, 2003) in a manner similar to the extraction of other high-level percepts, such as the detection of physical structure from motion (Scholl \& Tremoulet, 2000). Some authors have suggested that the perception of causality may even be served by a specialized cognitive module (Leslie \& Keeble, 1987; Scholl \& Nakayama, 2002). 
Evidence for causal perception is, however, complicated by the method used to assess the perceptual experience. Adult observers are typically asked to report whether they perceived the motion of one stimulus to cause the motion of another. These self-reports are open to the influence of postperceptual interpretation and may therefore reflect the outcome of inferential processes. Thus, evidence for causal perception from observer reports is confounded with causal inference. Support for this supposition comes from the observation that, contrary to Michotte's original contention, not all observers report perceptual causality on first encountering the stimuli (White, 1988), and reports of perceptual causality by adult observers are affected by contextual effects such as practice and prior exposure to the stimuli (Gruber, Fink, \& Damm, 1957; Powesland, 1959). Furthermore, sensitivity to causal structure has been shown to undergo subtle changes with infant development (Cohen, Amsel, Redford, \& Marianella, 1998), and reports from older children also show changes with age, suggesting that developing reasoning ability contributes to reports (Schlottmann, 1999, 2001; Schlottman, Allen, Linderoth, \& Hesketh, 2002). Although variance in observers' reports is potentially due to response bias effects and not to changes in the percept, these age- and experience-related changes highlight the difficulty with applying concepts of modularity to perceptual causality (Schlottmann, 2000).

The most successful attempt to date to separate causal perception from causal inference assessed whether a learned association between a predictive event (a color change) and movement of an object affected the perception of causality from launch events (Schlottmann \& Shanks, 1992). The impact of an object with another did not reliably predict the movement of the second object, but a color change of the second object did. Participants' ratings of the degree of perceived causality were not affected by the color change, suggesting that they were based not on predictive contingency but on the temporal properties of the collision. In a second task, participants were asked to judge the necessity of collisions for movement in a set of events that included a color change that predicted movement in collisions with or without temporal contiguity, as well as movements without a collision. These judgments were sensitive to the contingency between the color change and movement, but ratings of perceived causality were not, suggesting that these two aspects of understanding causality involve different processes.

Whereas perceptual causality is the term commonly used to describe a putative perceptual process that underlies participants' reporting of an impression of causality from dynamic displays, inferential causality describes the application of logical rules and conceptual knowledge to the interpretation of events. In an attempt to separate causal perception from causal inference in the brain, we tested 2 right-handed callosotomy (split-brain) patients (J.W. and V.P.). As visual input to the striate cortex is lateralized to the contralateral hemisphere, and only the anterior commissure and subcortical connections remain between the hemispheres, each hemisphere in the split brain can be tested in relative isolation. Previous testing of callosotomy patients has allowed seemingly indivisible cognitive processes to be separated into lateralized components. For example, Baynes, Eliassen, Lutsep, and Gazzaniga (1998) found that spoken and written language output can be controlled independently by the two disconnected hemispheres.
Testing each hemisphere in relative isolation may allow perceptual and inferential processes involved in understanding causality to be dissociated.

Repeated testing of callosotomized, or split-brain, patients has shown that the right hemisphere possesses an advantage for tasks that require visuospatial integration or discrimination (Corballis, 2003; Corballis, Funnell, \& Gazzaniga, 2002), such as mental rotation (Corballis \& Sergent, 1988) and perceiving illusory contours (Corballis, Fendrich, Shapley, \& Gazzaniga, 1999). Recent testing of 1 callosotomy patient included in this study (J.W.) found that the isolated right hemisphere also exhibited better performance than the left hemisphere in temporal-discrimination tasks with visually presented stimuli (Funnell, Corballis, \& Gazzaniga, 2003; Handy, Gazzaniga, \& Ivry, 2003). Other split-brain patients have shown a right hemispheric advantage for temporal discrimination of stimulus onset asynchronies in the range necessary to produce apparent movement (Forster, Corballis, \& Corballis, 2000).

Conversely, the left hemisphere has a greater ability to interpret complex stimuli and actions, including those of the right hemisphere (Gazzaniga, 2000). The left hemisphere often creates elaborate explanations for events and categorizes stimuli, in spite of the fact that this can interfere with performance on tasks that can be accomplished without the use of such elaboration. For example, in a simple task requiring the participant to predict which of two stimuli will appear on a given trial, the isolated left hemisphere of split-brain patients attempted to distribute its responses in a way that matched the probability with which each stimulus appeared. By contrast, the right hemisphere adopted the simpler strategy of always choosing the most frequently presented stimulus, thus maximizing the number of correct responses (Wolford, Miller, \& Gazzaniga, 2000). Thus, the left hemisphere pursued a suboptimal strategy that was based on an interpretation of the structure of the experimental context. This tendency to generate hypotheses about experimental structures and stimuli also has a deleterious effect on left-hemispheric memory performance. The left hemispheres of two split-brain patients performed at below chance on a task that required distinguishing previously presented items from novel items, whereas the right hemispheres performed well. The left hemispheres' poor performance was due to falsely recognizing novel pictures of scenes that were consistent with the common scenes presented for encoding (Phelps \& Gazzaniga, 1992).

The interpretive nature of the left hemisphere can be demonstrated by an experimental procedure in which two different scenes are presented separately to the two hemispheres of a split-brain patient. Each hemisphere is then required to choose, using the contralateral hand, an item from an array of pictures that is consistent with the scene presented to that hemisphere. When the patient is asked why he or she chose the item with his or her left hand (right hemisphere), the patient replies with an elaborative interpretation that is consistent with the scene presented to the patient's verbally able left hemisphere (Gazzaniga, 2000). Thus, the left hemisphere generates hypotheses about patterns in the environment, elaborates on information it receives, and creates explanations for events that include the actions of an isolated right hemisphere.

Although the brains of callosotomy patients differ greatly from intact brains, evidence from both behavioral testing (Funnell, Corballis, \& Gazzaniga, 1999; Funnell et al., 2003; Gazzaniga \& 
Smylie, 1983; Gazzaniga, Smylie, Baynes, Hirst, \& McClearly, 1984; Stone, Nisenson, Eliassen, \& Gazzaniga, 1996) and functional imaging studies (Cohen et al., 1996; Hugdahl, 2000; Richter, Ugurbil, Georgopoulos, \& Kim, 1997) suggests that the patterns of functional lateralization observed in the split brain are congruent with those found in neurologically intact participants in many different cognitive domains. As the callosotomy operation involves invasive surgery, and because split-brain patients have diverse medical histories, it is desirable to test as many patients as possible. This heterogeneity does, however, increase the impressiveness of obtaining the same result in 2 or more patients (Hellige, 1993).

Though patterns of lateralization derived from studies of patients and neurologically intact control participants are broadly consistent, behavioral asymmetries in neurologically intact participants are much smaller (Hellige, 1993). The corpus callosum allows many types of information to be transmitted between the hemispheres in only a few milliseconds (Zaidel \& Iacoboni, 2003), meaning that it is possible to test each hemisphere in relative isolation only when the corpus callosum is cut. The presence of an intact callosum thus masks the specific contributions of each hemisphere and reduces evidence for hemispheric asymmetries relative to those apparent in the divided brain.

On the basis of what is known of the relative abilities of the two hemispheres, we predicted that the right hemisphere would exhibit an advantage for accurately perceiving causal structure in displays of moving stimuli (see Experiment 1). Accurate performance on this task depends on the ability to determine the spatial and temporal contiguity of collision events. Conversely, we predicted that the performance of the left hemisphere would exceed that of the right hemisphere on a test of causal inference (see Experiment 2 ), as the left hemisphere has demonstrated superior interpretive ability to that of the right hemisphere. Animated examples of the stimuli for Experiments 1 and 2 are available for downloading; see the supplemental material on the Web at http://dx.doi.org/10.1037/ 0894-4105.19.5.591.supp.

If the expected right-hemispheric advantage on the test of perceptual causality is present in the absence of the ability to draw simple causal inference, this would constitute evidence for the involvement of the perceptual system in extracting causality from the interactions of moving objects. This finding, coupled with evidence for a left-hemispheric advantage in drawing simple causal inference but poor performance on the test of perceptual causality, would imply that perceptual causality and causal inference depend on different hemispheres of the brain and can be dissociated.

A perceptual process that depends on one hemisphere should be available to both hemispheres of the intact brain. Thus, we predicted that intact callosal transmission would allow neurologically intact participants to perform both tasks well regardless of the hemisphere to which the stimuli were presented.

\section{Experiment 1}

\section{Method}

Participants. Two callosotomy patients were tested. J.W. is a 50-yearold man, and V.P. is a 52-year-old woman. Both patients underwent staged section of the corpus callosum in 1979. The patients' presurgical medical histories are available elsewhere (Gazzaniga, Naas, Reeves, \& Roberts,
1984). As confirmed by MRI, the corpus callosum has been entirely severed in patient J.W. (Gazzaniga, Holtzman, Deck, \& Lee, 1985) and has been severed, except for a small cross section of fibers in the rostrum, in patient V.P. (Corballis, Inati, Funnell, Grafton, \& Gazzaniga, 2001).

Six neurologically intact participants were also tested. They were drawn from the pool of undergraduate students at Dartmouth College. All participants gave informed consent to the experiment and received either class credit or financial compensation for their involvement.

Materials. Stimuli were strictly lateralized to one visual field for all participants. This was achieved in a different manner for the 2 split-brain patients because of the necessity of testing at different times and locations. Lateralization ensured that stimuli were presented directly to only one hemisphere on each trial. When testing patient J.W., we minimized head movements using a head rest and bite bar. A dual-Purkinje-image eye tracker (Crane \& Steele, 1985) was used to monitor the eye movements, and a mirror deflector system was used to stabilize images on the retina (Crane \& Clark, 1978). The system has a resolution of approximately $1 \mathrm{~min}$ of arc and a response time of less than $2 \mathrm{~ms}$. J.W. viewed the display monocularly with his right eye through the stabilizer lens at an effective viewing distance of $63 \mathrm{~cm}$. Head movements were minimized for patient V.P. and for the neurologically intact participants with a chin and forehead rest. Eye position was monitored using an iViewX infrared eye tracker (SensoMotoric Instruments, Boston, MA). This system generated a transistor-to-transistor logic pulse within $30 \mathrm{~ms}$ (T. Dowe, personal communication, December 3,2003) when a saccade was made into either the left or right visual field. Stimuli were removed from the screen when this pulse was received by the computer controlling the experiment, thus preventing extended viewing. Deviations of eye position from the fixation point were recorded. V.P. and the neurologically intact participants viewed the stimulus display from a distance of $57 \mathrm{~cm}$.

Procedure. In the test of causal perception, collision events (see Figure 1) were presented to one hemisphere at a time by confining stimuli to one side of the visual field. Stimuli of this type have been used in many studies to assess the perception of causality from mechanical interactions between objects (see Scholl \& Tremoulet, 2000, for a review). In the causal condition, the impacted ball moved immediately after it was struck. The noncausal condition included either a spatial gap or temporal delay between movements of the two balls. Stimuli were presented within rectangular white patches superimposed on a black background and subtending approximately $8^{\circ}$ of horizontal visual angle. The white patches were displayed at locations to the left and right of fixation with the inner edge at approximately $5.5^{\circ}$. This location was sufficiently eccentric to avoid visual field overlap (Fendrich \& Gazzaniga, 1989) and to ensure that the stimuli were presented to only one hemisphere in the split brain. The visual field of presentation varied randomly between trials.

Stimuli comprised two circular black disks (representing balls) that were approximately $0.75^{\circ}$ in diameter. One stimulus was always located adjacent to either the left or right edge of the patch. The other stimulus was located near the middle of the patch, with one edge approximately $3^{\circ}$ from the other. Participants viewed three types of visual events, each lasting two seconds: causal, temporal delay, and spatial gap. The events moved from left to right and from right to left. For the causal event, the two black balls appeared motionless for $500 \mathrm{~ms}$, after which the first ball rolled horizontally across the screen and collided with the second ball. Immediately after
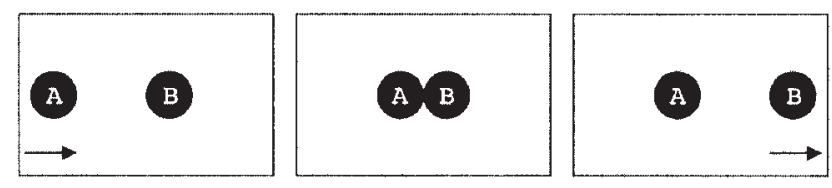

Figure 1. Stimuli for Experiment 1. Three panels depict the motion of a ball, $A$, toward another ball, $B$, and the subsequent motion of $B$. 
A.

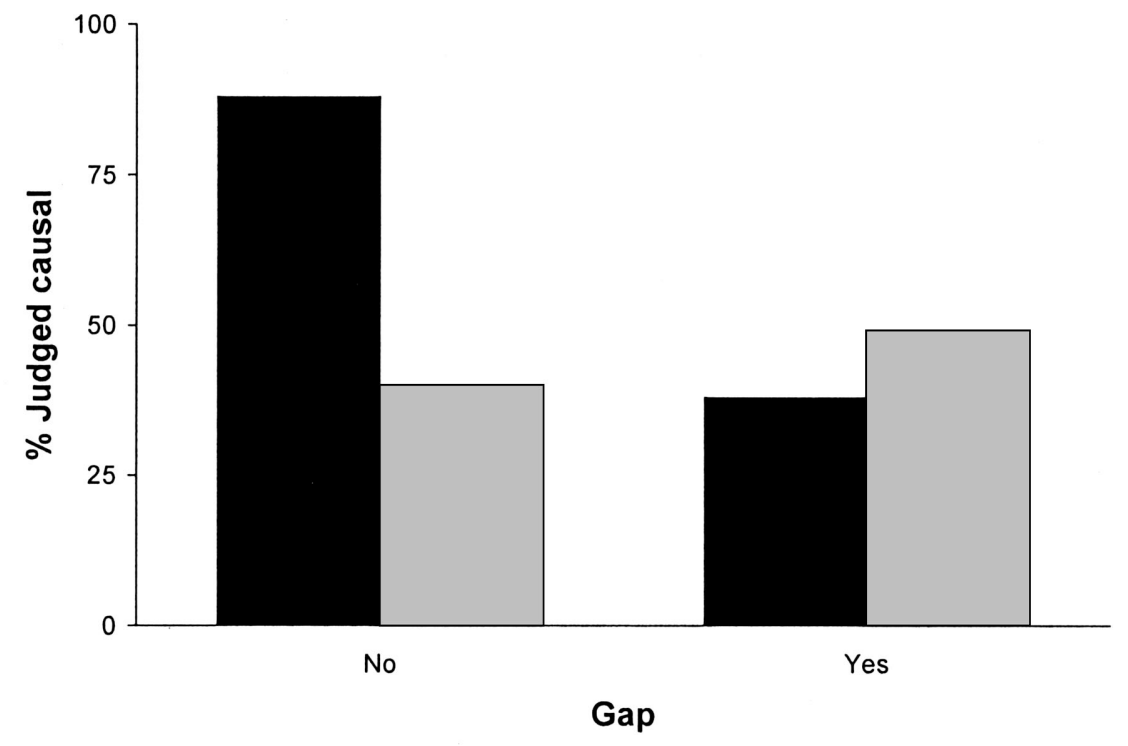

B.

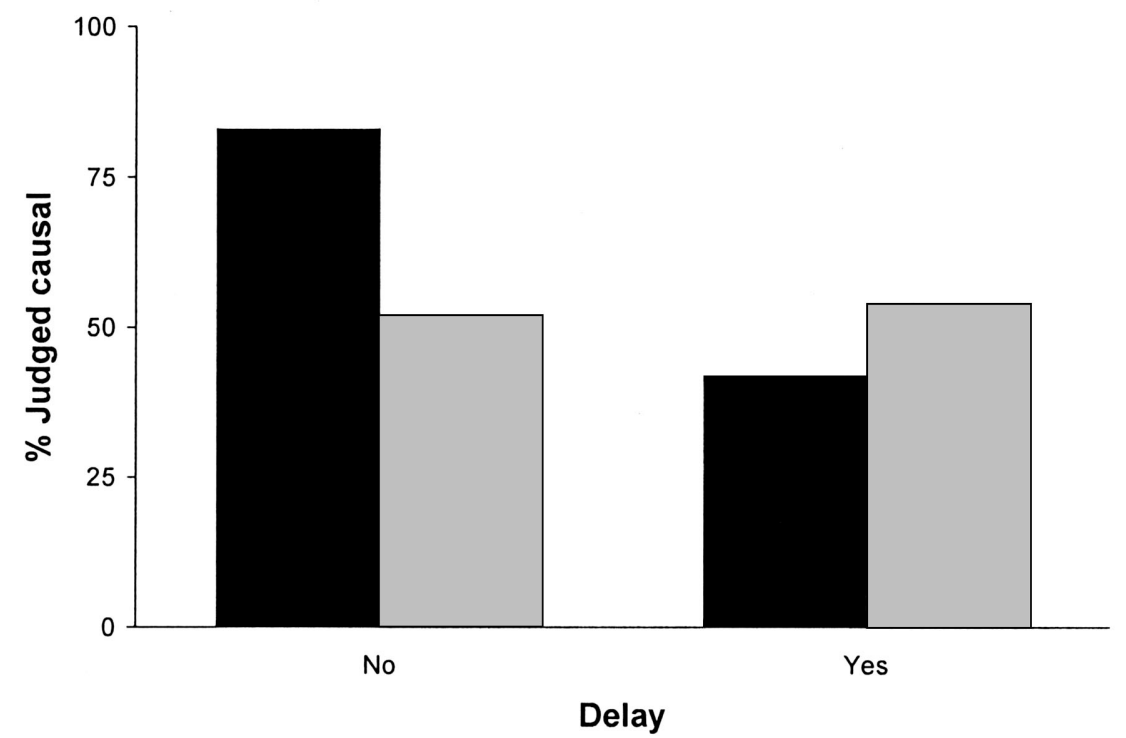

Figure 2. Results for Experiment 1. Percentage of trials in which stimulus interactions were judged causal as a function of visual field and hemisphere. The solid bar indicates left visual field, right hemisphere; the shaded bar indicates right visual field, left hemisphere. (A) patient J.W., spatial; (B) patient J.W., temporal; (C) patient V.P., spatial; (D) patient V.P., temporal.

the first ball made contact with the second ball, the second ball moved horizontally in the same direction, stopped at the edge of the patch, and lay motionless for $500 \mathrm{~ms}$. For the temporal delay events, the motion of the two balls was identical except for a delay between when the balls collided and when the second ball moved. The delay was either 30, 90, 150, or 240 $\mathrm{ms}$. For the spatial gap events, the first ball to move stopped before contacting the second ball, at which time the second ball moved in the same direction as the first. Gaps between the two stimuli were either $0.25,0.75,1.25$, or 2 stimulus diameters. These gaps were equivalent to the distance traveled by a moving stimulus in the durations used as delays for the temporal delay events. Thus, the magnitudes of the spatial and temporal manipulations were equated within the noncausal condition.

Participants were instructed to maintain fixation on a central point and respond by pressing one of two buttons with the hand on the same side as 
C.

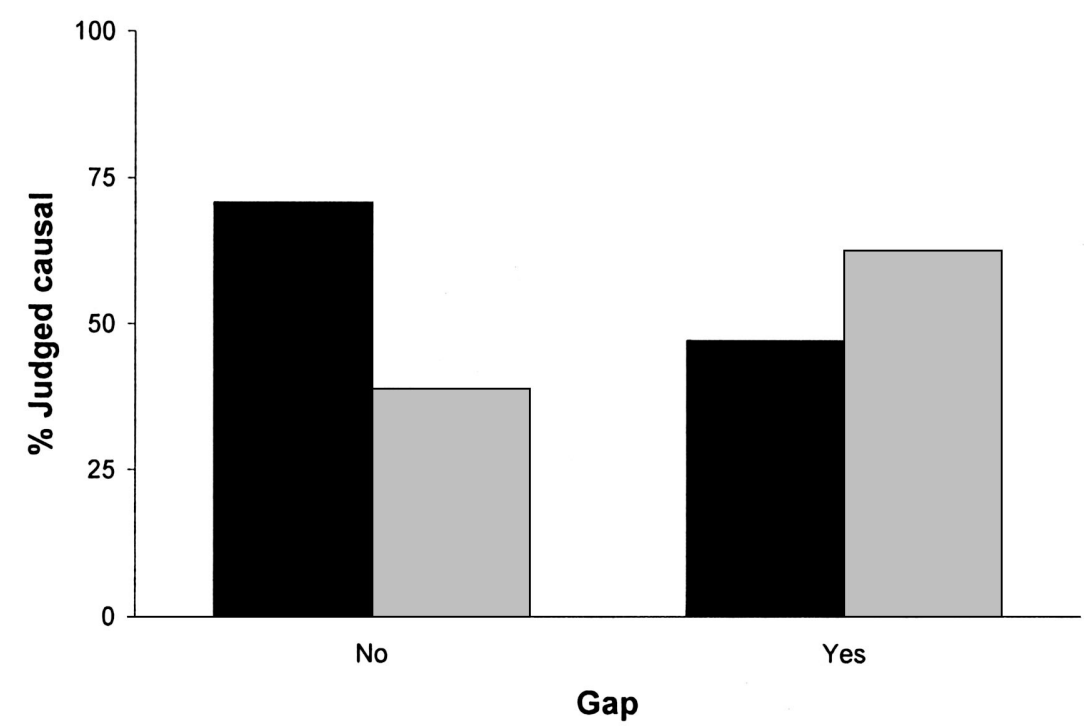

D.

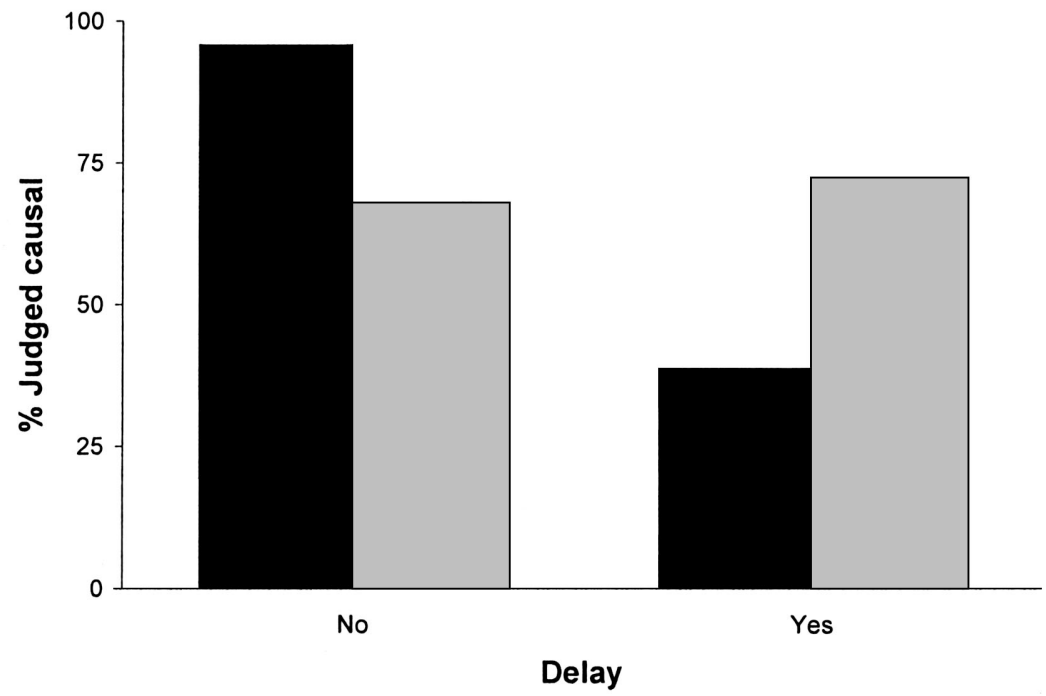

Figure 2. (continued).

the stimuli were displayed. These buttons were $K$ and $M$ with the right hand and $S$ and $X$ with the left hand. Participants were to press one button if it appeared that the first stimulus to move had caused the second stimulus to move and the other button if it appeared that the second stimulus to move had moved of its own accord. Participants were given a short familiarization run with the tasks, with the stimuli displayed in central vision. The experiment was run in blocks of 32 trials. Each block consisted half of causal events and half of either spatial gap or temporal delay events. Within each block, the direction of motion and the visual field of presentation were randomized. The 6 control participants completed four blocks of each of the two versions of the experiment (spatial and temporal). The order of the two versions was counterbalanced across participants. Patients J.W. and V.P. completed 19 blocks and 9 blocks, respectively, of each of the two versions over several days of testing.

\section{Results}

Both callosotomy participants showed the same pattern of results. The motion of the impacted ball was considerably more often reported as being caused by the motion of the first ball when collisions were contiguous (see Figure 2, A-D). However, this was true only of responses made by the right hemisphere. The presence 
of a gap or delay either made no difference to reports of causality by the left hemisphere or slightly increased the likelihood of reporting causality (as in graph $\mathrm{C}$ in Figure 2). The percentage of trials judged as causal are presented for all participants in Table 1. The percentages are broken down by visual field, type, and size of the incontiguity.

Individual analyses were performed for each of the patients to compare one hemisphere to the other. The effect of contiguity on the reported perception of causality was assessed as the interaction between the experimental condition (e.g., contiguous or incontiguous) and the response (e.g., causal or noncausal). This was calculated for each patient with the use of multidimensional chi-square statistics (Winer, 1971). The three-way interaction with visual field yielded a measure of differences between the two hemispheres in the effect of contiguity on response. The significant chi-square statistics for these three-way interactions are given in Table 2 for each callosotomy patient for the temporal delay and spatial gap manipulations. Thus, in both callosotomy patients, the perception of causality reported by the right hemisphere was significantly more affected by the contiguity of the two stimuli than was the perception reported by the left hemisphere.

The group of 6 callosally intact participants showed no difference between visual fields in the effect of incontiguity on ratings of causality. For these observers, contiguous collisions were rated as causal on over $95 \%$ of trials in each field. Stimuli that included a spatial incontiguity were rated as causal on $33 \%$ of trials in both visual fields. Stimuli that included a temporal incontiguity were rated as causal on $28 \%$ of trials in both visual fields. Seventy-six percent of these judgments of causality in the absence of contiguity were made when the delay or gap was of the smallest magnitude, $30 \mathrm{~ms}$ or 0.25 stimulus diameters.

\section{Discussion}

The participants' responses as to whether the first ball caused the second ball to move were determined primarily by spatial and temporal contiguity, as in earlier studies of perceptual causality in
Table 2

Multidimensional Chi-Square Statistics for Experiment 1 for the Interaction Between Condition, Response, and Visual Field

\begin{tabular}{ccc}
\hline & \multicolumn{2}{c}{ Contiguity manipulation } \\
\cline { 2 - 3 } Patient & Temporal delay & Spatial gap \\
\hline J.W. & $\chi^{2}(1, N=1)=25.29$, & $\chi^{2}(1, N=1)=49.80$, \\
$p<.001$ & $p .001$ \\
V.P. & $\chi^{2}(1, N=1)=26.89$, & $\chi^{2}(1, N=1)=20.78$, \\
& $p<.001$ & $p<.01$ \\
\hline
\end{tabular}

neurologically intact adults (Michotte, 1963; Schlottmann \& Shanks, 1992; Scholl \& Nakayama, 2002). The greater the incontiguity between the movements of the two stimuli, the less likely participants were to rate the collision as causal. This study extends this finding to situations in which stimuli are confined to one visual hemifield in the intact brain.

Of importance, in the test of perceptual causality, a pattern of responding to collision stimuli similar to that seen in the neurologically intact participants was obtained only from the right hemisphere of callosotomy patients J.W. and V.P. Unlike those of neurologically intact participants, the left hemispheres of both patients did not use the spatiotemporal properties of an interaction between moving objects to determine whether the interaction was causal. In Experiment 2, we investigated whether the hemispheres differ in their ability to infer causality from patterns of dependence and independence between actions and their effects.

\section{Experiment 2}

The test of causal inference was adapted from a task used with children (Gopnik, Sobel, Schulz, \& Glymour, 2001). Gopnik et al. (2001) presented children of ages 2, 3, and 4 years old with causal relations between objects (some of which were known as blickets) and a machine that lit up and played music when a blicket was

Table 1

Percentage of Trials Rated as Causal for All Participants for Each Visual Field, Contiguity Manipulation, and Size of Incontiguity

\begin{tabular}{|c|c|c|c|c|c|c|c|c|c|c|}
\hline \multirow[b]{3}{*}{ Participant } & \multicolumn{10}{|c|}{ Gap (stimulus diameters) } \\
\hline & \multicolumn{5}{|c|}{ LVF } & \multicolumn{5}{|c|}{ RVF } \\
\hline & 0 & 0.25 & 0.75 & 1.25 & 2.0 & 0 & 0.25 & 0.75 & 1.25 & 2.0 \\
\hline Control & 96 & 83 & 31 & 10 & 8 & 97 & 84 & 31 & 10 & 4 \\
\hline J.W. & 88 & 66 & 45 & 24 & 21 & 41 & 42 & 55 & 47 & 53 \\
\hline \multirow[t]{4}{*}{ V.P. } & 71 & 72 & 44 & 44 & 17 & 39 & 50 & 66 & 55 & 78 \\
\hline & \multicolumn{10}{|c|}{ Delay (ms) } \\
\hline & \multicolumn{5}{|c|}{ LVF } & \multicolumn{5}{|c|}{ RVF } \\
\hline & 0 & 30 & 90 & 150 & 240 & 0 & 30 & 90 & 150 & 240 \\
\hline Control & 96 & 83 & 31 & 10 & 8 & 95 & 94 & 13 & 2 & 2 \\
\hline J.W. & 84 & 84 & 45 & 29 & 24 & 63 & 61 & 61 & 71 & 68 \\
\hline V.P. & 96 & 100 & 50 & 0 & 6 & 68 & 89 & 67 & 78 & 56 \\
\hline
\end{tabular}

Note. $\quad \mathrm{LVF}=$ left visual field, right hemisphere; RVF = right visual field, left hemisphere; Gap = spatial gap between stimuli; Delay $=$ temporal delay between stimulus movements. 
placed on it. In Gopnik et al.'s "one-cause" task, children saw one object being placed on the machine, which activated the machine, followed by another object that did not activate the machine. On two occasions both objects were placed on the machine, which activated. The children were then asked whether each of the objects was a blicket, that is, an object that would activate the machine. Gopnik and colleagues found that children of all three age groups took patterns of variation and covariation into account when deciding whether an object was a blicket. On the basis of the results of this study and additional experiments, the authors concluded that even very young children can infer causality in object interactions from patterns of dependence and independence of actions and effects. This finding suggests that young children acquire new causal maps or abstract coherent representations of causal relations among events. The exact manner in which this acquisition takes place is a matter of recent debate, as a number of theoretical models can account for causal learning based on observed statistical regularities (Gopnik et al., 2001; Novick \& Cheng, 2004; Shanks, 1995). The theoretical implications of this experiment are discussed in depth in Gopnik et al. (2004). The present study was the logical equivalent of Gopnik et al.'s (2001) one-cause experiment and assessed whether each hemisphere of the divided brain could infer causality from patterns of actions and effects.

\section{Method}

Participants. The same 2 callosotomy patients and 6 neurologically intact participants who took part in Experiment 1 also completed Experiment 2.

Materials. Stimuli were lateralized to only one visual field on each trial. The same eye-tracking methods used in Experiment 1 were also used in Experiment 2. The only difference between experiments was that V.P. viewed the stimuli in Experiment 2 from a distance of $47 \mathrm{~cm}$. Stimuli were displayed at an eccentricity of approximately $3.5^{\circ}$ for J.W. and the neurologically intact participants and at $4.5^{\circ}$ for V.P.

Procedure. Participants viewed a series of movements of "switches" and their effect on a "lightbox" (see Figure 3) and then had to decide whether a presented stimulus (one of the switches) was the cause of the box's illumination. The lightbox (large square) and two switches (red and green) were presented four times on each trial. Arrows in Figure 3 indicate four sequential movements of the switches, and the corresponding state of the lightbox is depicted as either on (white) or off (gray). Movement of

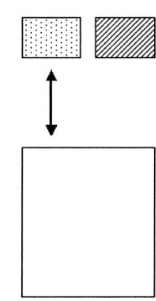

1

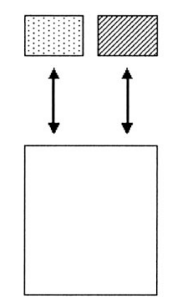

2

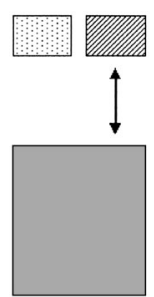

3

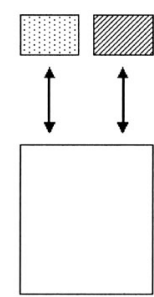

4

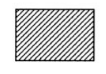

Probe
Figure 3. Stimuli for Experiment 2. The sequential presentation of four stimulus interactions and a response probe are shown, representing one trial. Arrows indicate the movement of one or both of the colored "switches" on each presentation. Dot-filled boxes indicate a green switch; boxes with diagonal lines indicate a red switch. The shaded box on presentation 3 indicates a lack of illumination of the lightbox on that presentation. each switch was paired with the light coming on more often than with the light staying off, but only one switch was paired with the light coming on independently of the movement of the other switch. Each movement of one or both switches lasted $1 \mathrm{~s}$. One switch was then presented at the end of the trial (probe), and participants had to respond with a two-choice button press as to whether that switch had turned on the light. Within each block of 16 trials, the color of the switch that activated the light, the visual field of presentation, and the relative location of the two switches were randomized. Responses were made with the hand on the same side of the body as the stimuli were displayed. The 6 control participants completed four blocks of the experiment. Patients J.W. and V.P. completed 16 and 17 blocks, respectively, over several days of testing.

\section{Results}

Only the left hemisphere of the 2 split-brain patients showed sensitivity to the nature of the response probe. When the response probe represented the cause of the effect, J.W. and V.P. were more likely to respond in the affirmative, but only with responses made by the left hemisphere (see Figure 4). The percentage of correct responses is shown for each patient and each visual field in Figure 5. Thus, only the left hemisphere was able to correctly determine the causal nature of the presented stimulus on the basis of the effect of its movement on another stimulus. For V.P., the nature of the response probe had very little effect on responses made by the right hemisphere. J.W. actually responded in the affirmative to a greater proportion of noncausal probes than to causal probes presented to the right hemisphere. The three-way interaction, assessed separately for each patient by multidimensional chi-square statistics, between probe type (causal or noncausal), response, and visual field, was significant in both cases: J.W., $\chi^{2}(1, N=$ $1)=50.77, p<.01$, and V.P., $\chi^{2}(1, N=1)=25.20, p<.01$.

The 6 callosally intact participants showed no difference between visual fields in ability to determine the cause. Performance was near perfect (98\% correct in both fields) for these participants.

\section{General Discussion}

We found a double dissociation between the performances of the two hemispheres on the two tasks for both of the callosotomy patients. Both patients' left hemispheres were able to draw simple causal inference but were unable to use this capacity to determine the causal nature of collision events. Conversely, both patients' right hemispheres were sensitive to the causal nature of collision events but were unable to draw simple causal inference. Together, these results suggest that understanding causality involves multiple processes and provide support for the existence of dissociable perceptual and inferential components. The right hemisphere is specialized for the fine-grained analysis of interactions between moving stimuli and for extracting causal structure from such events. The isolated left hemisphere is unable to resolve the finer spatiotemporal properties of a fast-moving stimulus, but is able to infer causality from contingencies between events, such as the movement of a switch and effects such as the onset of a light. Thus, inferring causality over longer time frames and more complex sets of events is supported by the left hemisphere. Our data show that the perception of causality can be present in a hemisphere of the brain that is unable to draw simple causal inference. Thus, inference at a level of sophistication reached by 2 -year-olds is not necessary for normal performance with collision stimuli. 
A.

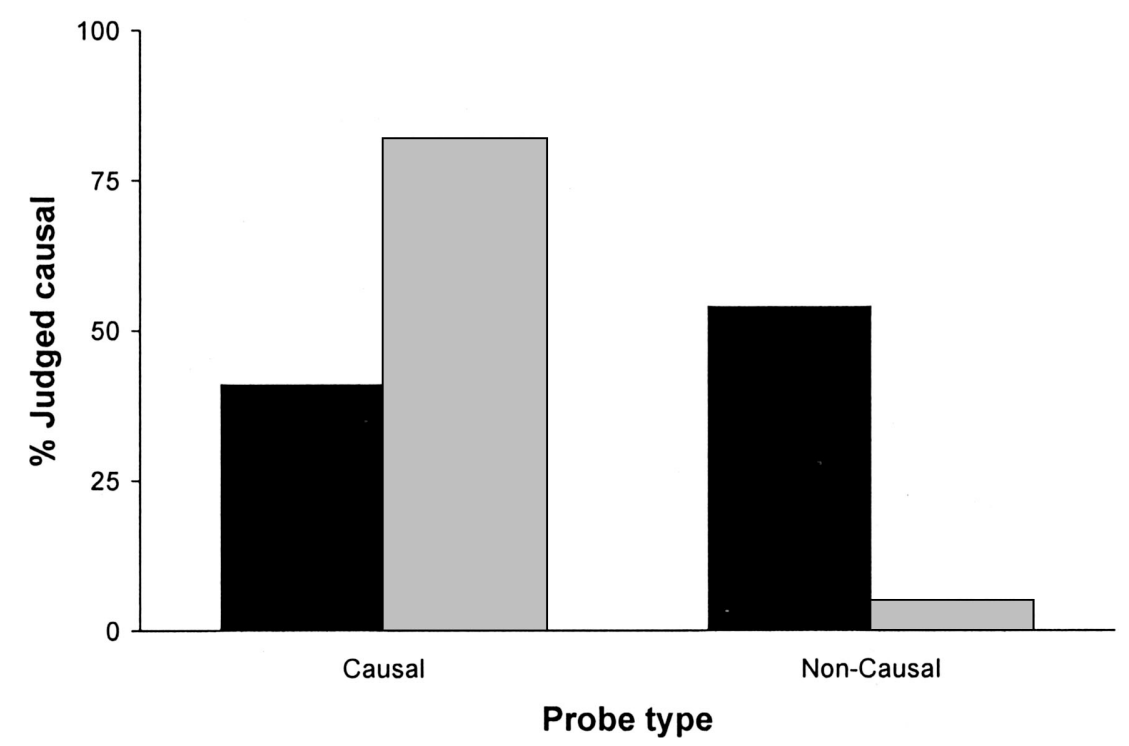

B.

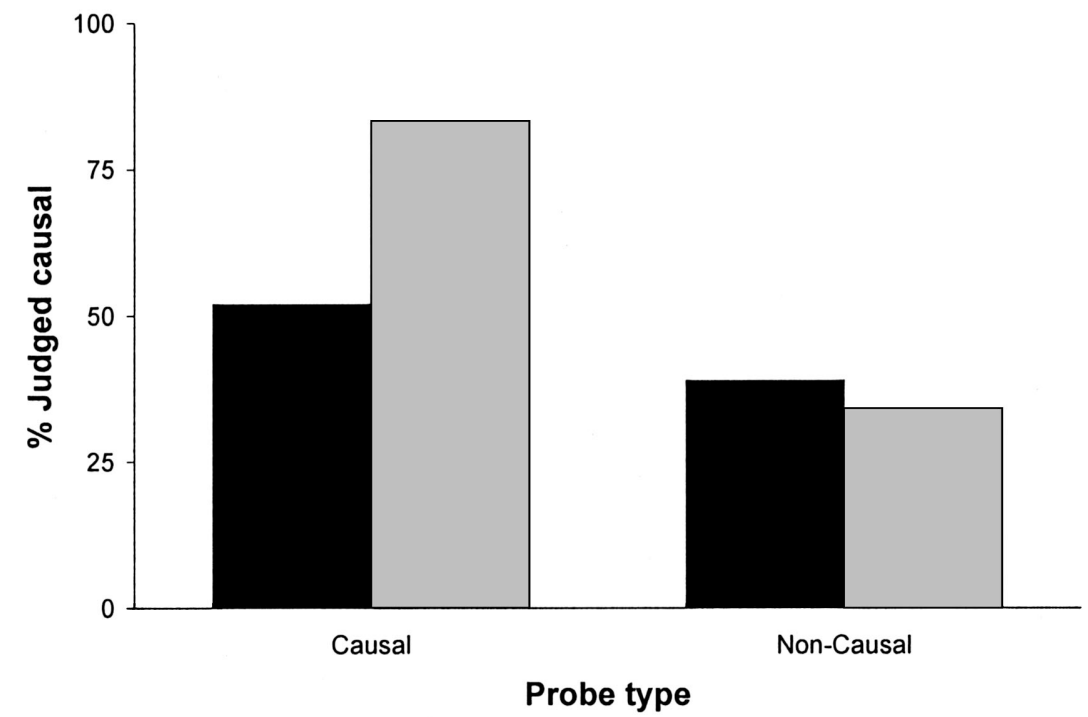

Figure 4. Results for Experiment 2. Percentage of trials in which the patient responded causal by probe type (causal or noncausal). The solid bar indicates left visual field, right hemisphere; the shaded bar indicates right visual field, left hemisphere. (A) patient J.W., (B) patient V.P. 
A.

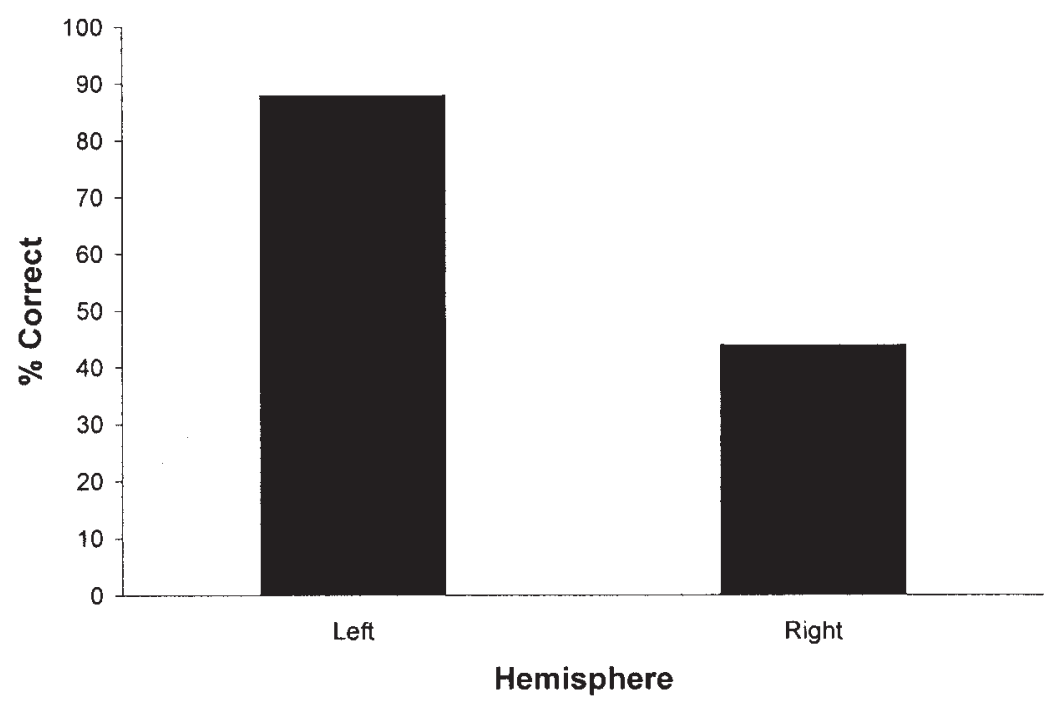

B.

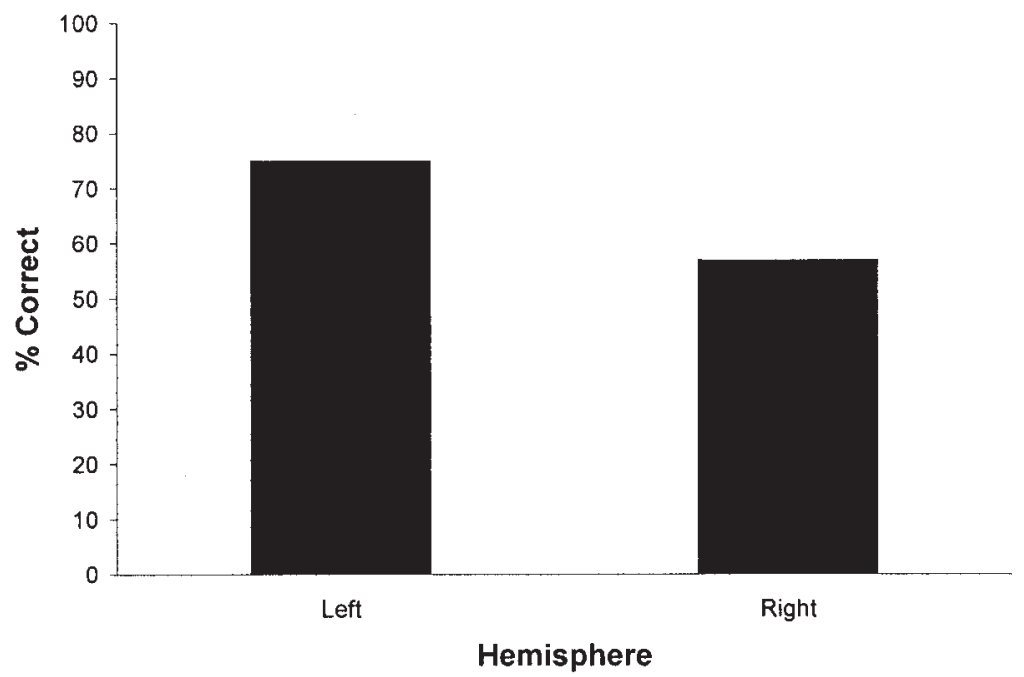

Figure 5. Results for Experiment 2. Percentage of correct responses by hemisphere. (A) patient J.W., (B) patient V.P.

The observation of a double dissociation between performances on the two tasks implies that the ability to draw inference is not sufficient for determining the causal structure of dynamic events. If responses in Experiment 1 were determined solely by an inferential process, then the left hemisphere should have been able to perform at above chance in Experiment 1, which would logically involve a much simpler inference than that required for Experiment 2. Instead, the poor performance of the left hemispheres of the 2 patients implies that something more is required. This righthemispheric process, which extracts the causal structure of dynamic events, is likely to be similar to perceptual grouping processes for which the right hemisphere has a demonstrated superiority over the left (Corballis, et al., 1999).

An alternative explanation for the inability of the isolated right hemispheres to perform above chance in Experiment 2 is that the two hemispheres differ in working memory capacity, with only the 
left hemisphere capable of maintaining a representation of the four sequentially presented stimuli long enough to determine the correct response. Several lines of research suggest, however, that the present results are not due to a working memory capacity difference between the hemispheres.

Both hemispheres in the intact brain demonstrate involvement in working memory tasks, as activation is typically observed bilaterally in functional MRI (fMRI) studies, although some studies have reported greater right-hemispheric activation in spatial paradigms and greater left activation in nonspatial paradigms (Manoach et al., 2004; see D'Esposito et al., 1998, for a review).

Most relevant to interpretation of the present study are tasks involving split-brain patients V.P. and J.W. Both patients show comparable working memory performance with the two hemispheres. In a study in which stimuli were serially presented at four locations within a grid, J.W. showed no difference between the two hemispheres in ability to determine whether a probe sequence matched that of a target sequence (Holtzman \& Gazzaniga, 1985). These data support the hypothesis that each hemisphere is capable of maintaining a representation of the order of stimulation of four spatial locations over a period of $5.7 \mathrm{~s}$. Similarly, in a study in which shapes were serially presented to each visual field, each hemisphere of V.P.'s brain was able to maintain the identities of three shapes over approximately $2.5 \mathrm{~s}$ and then decide whether a briefly presented probe had been included in the maintained set (Holtzman \& Gazzaniga, 1982). Studies with split-brain monkeys have also shown that both isolated hemispheres are able to maintain a representation of serially presented stimuli (Lewine, Doty, Astur, \& Provencal, 1994).

Thus, the pattern of results observed in the present study is best explained by a model in which each of the hemispheres contributes its specialized competency to determining causality rather than by a hemispheric difference in working memory. ${ }^{1}$ A right-hemispheric perceptual-grouping process that parses stimulus movements into a coherent assembly allows causal structure to be determined from rapid dynamic events. A left-hemispheric process that infers causality from patterns of covariation between events allows for the determination of causality in more complex situations.

This complementary division of processing between the hemispheres is an efficient use of cortical space because it reduces unnecessary duplication of function between the hemispheres (Nottebaum, 1977) and neuronal conduction time, as short (e.g., within-hemisphere) connections are more efficient than long (e.g., between-hemispheres) connections (Ringo, Doty, Demeter, \& Simard, 1994). These lateralized systems are likely integrated by the corpus callosum in the intact brain, allowing both systems to contribute to our understanding of causality. The neurologically intact participants were able to perform both tasks well regardless of to which hemisphere the stimuli were initially presented. ${ }^{2}$

Intact callosal transfer may account for differences between the lateralization of function suggested by the present study and that found by a recent fMRI investigation of causal structure in collision events. Blakemore et al. (2001) compared contiguous (causal) collision events with events in which there was no collision with and subsequent movement of a second stimulus. Blakemore et al.'s study showed bilateral activation in medial-temporal areas (MT/ V5) and the superior temporal sulcus and activation at the border of the intraparietal sulcus and angular gyrus of the left hemisphere. These activations were not affected by attention to the presence of causality, which was manipulated by requiring participants to report either perceived causality or the direction of motion. In a later reanalysis of the data, Fonlupt (2003) found that bilateral activation in the superior frontal sulcus was greater when participants made judgments about causality compared with when they attended to the direction of motion. These activations were not affected by the nature of the stimuli (causal or noncausal), and there was no clear lateralization of function at the group level. Activation was located in the right hemisphere of 4 participants, in the left hemisphere of 1 participant, and bilaterally in 3 participants. Callosal transfer may allow processes that depend critically on one hemisphere to nonetheless activate regions in the opposite hemisphere. For example, the right hemisphere is thought to be specialized for spatial processing, as this capacity is adversely affected by damage to the right parietal lobe. In a recent fMRI study of spatial processing, researchers found bilateral parietal lobe activation, but a detailed analysis revealed that the right hemisphere had a dominant role in "kick-starting" the process $(\mathrm{Ng}$ et al., 2000). Thus, bilateral activation may result from the initiation of processes by one hemisphere.

The integration of perceptual and inferential processes may be illuminated, in future research, by optical imaging of infants' brains and fMRI imaging of the brains of children. Cohen et al. (1998) have shown that the sensitivity of infants to causal structure changes over the course of the 1st year of life. Schlottmann (1999, 2001) and Schlottmann et al. (2002) have shown that the early sensitivity to perceptual causality is integrated with developing reasoning abilities over the course of the 1 st decade. Imaging the brains of children at different stages of their development may reveal the gradual onset of left-lateralized processes supporting reasoning, which become integrated with previously established processes supporting perceptual abilities, such as extracting causal structure. In the adult brain, it is only when normal hemispheric communication is disrupted, as in callosotomy, that the lateralized nature of the processes supporting causal perception and causal inference becomes observable.

\footnotetext{
${ }^{1}$ A simpler version of the inference task was also run with patient J.W. On 80 trials in each visual field, only the two single switch movements were presented. The working memory requirements for this task were, therefore, reduced relative to the task used in Experiment 2, but a similar result was produced. J.W.'s left hemisphere performed well at identifying the causal nature of the response probe $(98 \%$ correct), but his right hemisphere performed at chance ( $51 \%$ correct).

${ }^{2}$ Although the mean age (approximately 20 years) of the neurologically intact participants was younger than that of the 2 patients, this difference cannot account for the pattern of results observed in this study. Aging has been shown to detrimentally affect hemispheric transmission of sensorimotor information in callosally intact participants (Reuter-Lorenz \& Stanczak, 2000), but these effects are much smaller than the radical disconnection affected by severing the corpus callosum. The ages of participants included in the Reuter-Lorenz and Stanczak (2000) study were also much older (65-75 years) than the age of the 2 callosotomy patients ( 50 and 52 years). Thus, for both patients, the striking double-dissociation of the performance of the two hemispheres on the two tasks is highly unlikely to be the result of age, but is likely due to the surgical disconnection of the two hemispheres.
} 


\section{References}

Baynes, K., Eliassen, J. C., Lutsep, H. L., \& Gazzaniga, M. S. (1998, May 8). Modular organization of cognitive systems masked by interhemispheric integration. Science, 280, 902-905.

Blakemore, S. J., Fonlupt, P., Pachot-Clouard, M., Darmon, C., Boyer, P., Meltzoff, A. N., et al. (2001). How the brain perceives causality: An event-related fMRI study. NeuroReport, 12, 3741-3746.

Cohen, L. B., Amsel, G., Redford, M. A., \& Marianella, C. (1998). The development of infant causal perception. In A. Slater (Ed.), Perceptual development: Visual, auditory and speech perception in infancy (pp. 167-209). Hove, England: Psychology Press.

Cohen, M. S., Kosslyn, S. M., Breiter, H. C., DiGirolamo, G. J., Thompson, W. L., Anderson, A. K., et al. (1996). Changes in cortical activity during mental rotation. A mapping study using functional MRI. Brain, 119, 89-100.

Corballis, M. C., \& Sergent, J. (1988). Imagery in a commissurotomized patient. Neuropsychologia, 26, 13-26.

Corballis, P. M. (2003). Visuospatial processing and the right-hemisphere interpreter. Brain and Cognition, 53, 171-176.

Corballis, P. M., Fendrich, R., Shapley, R. M., \& Gazzaniga, M. S. (1999). Illusory contour perception and amodal boundary completion: Evidence of a dissociation following callosotomy. Journal of Cognitive Neuroscience, 11, 459-466.

Corballis, P. M., Funnell, M. G., \& Gazzaniga, M. S. (2002). Hemispheric asymmetries for simple visual judgments in the split brain. Neuropsychologia, 40, 401-410.

Corballis, P. M., Inati, S., Funnell, M. G., Grafton, S. T., \& Gazzaniga, M. S. (2001). MRI assessment of spared fibers following callosotomy: A second look. Neurology, 57, 1345-1346.

Crane, H. D., \& Clark, M. R. (1978). Three-dimensional visual stimulus deflector. Applied Optics, 17, 706-714.

Crane, H. D., \& Steele, C. M. (1985). Generation-V dual Purkinje image eyetracker. Applied Optics, 24, 524-537.

D'Esposito, M., Aguirre, G. K., Zarahn, E., Ballard, D., Shin, R. K., \& Lease, J. (1998). Functional MRI studies of spatial and nonspatial working memory. Cognitive Brain Research, 7, 1-13.

Fendrich, R., \& Gazzaniga, M. S. (1989). Evidence of foveal splitting in a commissurotomy patient. Neuropsychologia, 27, 273-281.

Fonlupt, P. (2003). Perception and judgement of physical causality involve different brain structures. Cognitive Brain Research, 17, 248-254.

Forster, B., Corballis, P. M., \& Corballis, M. C. (2000). Effect of luminance on successiveness discrimination in the absence of the corpus callosum. Neuropsychologia, 38, 441-450.

Funnell, M. G., Corballis, P. M., \& Gazzaniga, M. S. (1999). A deficit in perceptual matching in the left hemisphere of a callosotomy patient. Neuropsychologia, 37, 1143-1154.

Funnell, M. G., Corballis, P. M., \& Gazzaniga, M. S. (2003). Temporal discrimination in the split brain. Brain and Cognition, 53, 218-222.

Gazzaniga, M. S. (2000). Cerebral specialization and interhemispheric communication: Does the corpus callosum enable the human condition? Brain, 123, 1293-1326.

Gazzaniga, M. S., Holtzman, J. D., Deck, M. D. F., \& Lee, B. C. P. (1985). MRI assessment of human callosal surgery with neuropsychological correlates. Neurology, 35, 1763-1766.

Gazzaniga, M. S., Naas, R., Reeves, A., \& Roberts, D. (1984). Neurologic perspectives on right hemisphere language following surgical section of the corpus callosum. Seminars in Neurology, 4, 126-135.

Gazzaniga, M. S., \& Smylie, C. S. (1983). Facial recognition and brain asymmetries: Clues to underlying mechanisms. Annals of Neurology, 13, $536-540$.

Gazzaniga, M. S., Smylie, C. S., Baynes, K., Hirst, W., \& McClearly, C. (1984). Profiles of right hemisphere language and speech following brain bisection. Brain and Language, 22, 206-220.
Gopnik, A., Glymour, C., Sobel, D. M., Schulz, L. E., Kushnir, T., \& Danks, D. (2004). A theory of causal learning in children: Causal maps and Bayes nets. Psychological Review, 111, 3-32.

Gopnik, A., Sobel, D. M., Schulz, L. E., \& Glymour, C. (2001). Causal learning mechanisms in very young children: Two-, three-, and fouryear-olds infer causal relations from patterns of variation and covariation. Developmental Psychology, 37, 620-629.

Gruber, H. E., Fink, C. D., \& Damm, D. (1957). Effects of experience on perception of causality. Journal of Experimental Psychology, 53, 89-93.

Handy, T. C., Gazzaniga, M. S., \& Ivry, R. B. (2003). Cortical and subcortical contributions to the representation of temporal information. Neuropsychologia, 41, 1461-1473.

Hecht, H. (1996). Heuristics and invariants in dynamic event perception: Immunized concepts or non-statements. Psychonomic Bulletin and Review, 3, 61-70.

Hellige, J. B. (1993). Hemispheric asymmetry: What's right and what's left (pp. 9-24). Cambridge, MA: Harvard University Press.

Holtzman, J. D., \& Gazzaniga, M. S. (1982, December 24). Dual task interactions due exclusively to limits in processing resources. Science, $218,1325-1327$.

Holtzman, J. D., \& Gazzaniga, M. S. (1985). Enhanced dual task performance following corpus commissurotomy in humans. Neuropsychologia, 23, 315-321.

Hugdahl, K. (2000). Lateralization of cognitive processes in the brain. Acta Psychologica, 105, 211-235.

Hume, D. (1978). A treatise of human nature. Oxford, England: Oxford University Press.

Johnson-Frey, S. H. (2003). What's so special about human tool use? Neuron, 39, 201-204.

Leslie, A. M., \& Keeble, S. (1987). Do six-month-old infants perceive causality? Cognition, 25, 265-288.

Lewine, J. D., Doty, R. W., Astur, R. S., \& Provencal, S. L. (1994). Role of the forebrain commissures in bihemispheric mnemonic integration in macaques. Journal of Neuroscience, 14, 2515-2530.

McClure, J. (1998). Discounting causes of behavior: Are two reasons better than one? Journal of Personality and Social Psychology, 74, 7-20.

Manoach, D. S., White, N. S., Lindgren, K. A., Heckers, S., Coleman, M. J., Dubal, S., \& Holzman, P. S. (2004). Hemispheric specialization of the lateral prefrontal cortex for strategic processing during spatial and shape working memory. NeuroImage, 21, 894-903.

Michotte, A. (1963). The perception of causality (T. Miles \& E. Miles, Trans.). New York: Basic Books. (Original work published 1946)

Ng, V. W. K., Eslinger, P. J., Williams, S. C. R., Brammer, M. J., Bullmore, E. T., Andrew, C. M., et al. (2000). Hemispheric preference in visuospatial processing: A complementary approach with fMRI and lesion studies. Human Brain Mapping, 10, 80-86.

Nottebaum, F. (1977). Asymmetries in neural control of vocalization in the canary. In S. Harnad, R. W. Doty, L. Goldstein, J. Jaynes, \& G. Krauthamer (Eds.), Lateralization of the nervous system (pp. 23-44). New York: Academic Press.

Novick, L., \& Cheng, P. (2004). Assessing interactive causal inference. Psychological Review, 111, 455-485.

Oakes, L. M. (1994). Development of infants' use of continuity cues in their perception of causality. Developmental Psychology, 30, 869-870.

Phelps, E. A., \& Gazzaniga, M. S. (1992). Hemispheric differences in mnemonic processing: The effects of left hemisphere interpretation. Neuropsychologia, 30, 293-297.

Powesland, P. F. (1959). The effect of practice upon the perception of causality. Canadian Journal of Psychology, 13, 155-168.

Reuter-Lorenz, P. A., \& Stanczak, L. (2000). Differential effects of aging on the functions of the corpus callosum. Developmental Neuropsychology, 18, 113-137.

Richter, W., Ugurbil, K., Georgopoulos, A., \& Kim, S. G. (1997). Timeresolved fMRI of mental rotation. NeuroReport, 8, 3697-3702. 
Ringo, J. L., Doty, R. W., Demeter, S., \& Simard, P. Y. (1994). Time is of the essence-a conjecture that hemispheric specialization arises from interhemispheric conduction delay. Cerebral Cortex, 4, 331-343.

Schlottmann, A. (1999). Seeing it happen and knowing how it works: How children understand the relation between perceptual causality and underlying mechanism. Developmental Psychology, 35, 303-317.

Schlottmann, A. (2000). Is perception of causality modular? Trends in Cognitive Science, 4, 441-442.

Schlottmann, A. (2001). Perception versus knowledge of cause and effect in children: When seeing is believing. Current Directions in Psychological Science, 10, 111-115.

Schlottmann, A., Allen, D., Linderoth, C., \& Hesketh, S. (2002). Perceptual causality in children. Child Development, 73, 1656-1677.

Schlottmann, A., \& Shanks, D. R. (1992). Evidence for a distinction between judged and perceived causality. Quarterly Journal of Experimental Psychology: Human Experimental Psychology, 44(A), 321-342.

Scholl, B. J., \& Nakayama, K. (2002). Causal capture: Contextual effects on the perception of collision events. Psychological Science, 13, 493498.

Scholl, B. J., \& Tremoulet, P. D. (2000). Perceptual causality and animacy. Trends in Cognitive Science, 4, 299-309.

Shanks, D. R. (1995). The psychology of associative learning. Cambridge, England: Cambridge University Press.
Stone, V. E., Nisenson, L., Eliassen, J. C., \& Gazzaniga, M. S. (1996). Left hemisphere representations of emotional facial expressions. Neuropsychologia, 34, 23-29.

White, P. A. (1988). Causal processing: Origins and development. Psychological Bulletin, 104, 36-52.

White, P. A. (1995). The understanding of causation and the production of action. Hillsdale, NJ: Erlbaum.

Winer, B. J. (1971). Statistical principles in experimental design (2nd ed.). New York: McGraw-Hill.

Wolford, G., Miller, M. B., \& Gazzaniga, M. S. (2000). The left hemisphere's role in hypothesis formation. Journal of Neuroscience, 20, RC64(1-4).

Zaidel, E., \& Iacoboni, M. (2003). Does the CUD in SRT measure IHTT? Or: Is the crossed-uncrossed difference in the simple reaction time task a pure measure of interhemispheric transfer time? In E. Zaidel \& M. Iacoboni (Eds.), The parallel brain (pp. 259-266). Cambridge, MA: MIT Press.

Received December 26, 2003

Revision received September 23, 2004

Accepted October 25, 2004 